\title{
Urosepsis caused by Kluyvera Ascorbata in a Pregnant Woman
}

\author{
Akif Erbin1, Hasan Tahsin Gozdas², Yavuz Guler³ and Halil Lutfi Canat4 \\ ${ }^{1}$ Department of Urology, Haseki Training and Research Hospital, Istanbul, Turkey \\ 2 Department of Infectious Disease, Abant Izzet Baysal University, Bolu, Turkey \\ ${ }^{3}$ Department of Urology, Safa Hospital, Istanbul, Turkey \\ ${ }^{4}$ Department of Urology, Okmeydani Training and Research Hospital, Istanbul, Turkey
}

\begin{abstract}
Among the Kluyvera species, $K$. ascorbata has been isolated from only a few adult cases. Furthermore, there is little or no information in the literature as to whether the species of Kluyvera can cause a clinically significant infection in pregnant women. We report a case of urosepsis caused by K. ascorbata in a 23-year pregnant woman at 26 weeks of gestation who presented with left flank pain. Ultrasonography showed left grade 3 hydronephrosis, ureteral dilatation, and a 10-mm distal ureteral stone. The patient underwent laser lithotripsy and JJ placement. Ten days later, she was readmitted with urosepsis and empirical antibiotherapy and aggressive hydration were initiated. On the third day, $K$. ascorbata growth was detected in the urine culture. Based on the clinical status of the patient and the antimicrobial susceptibility testing, the treatment was switched to ertapenem $1 \times 1 \mathrm{~g} /$ day and was continued for 14 days. Among the Kluyvera species, $K$. ascorbata is the most frequent pathogen which may be isolated from pregnant women and can cause urosepsis. To the best of authors' knowledge, this is the first report showing the isolation of $K$. ascorbata in a pregnant woman which caused urosepsis.
\end{abstract}

Key Words: Kluyvera ascorbata, Pregnancy, Urosepsis.

How to cite this article: Erbin A, Gozdas HT, Guler Y, Canat HL. Urosepsis caused by Kluyvera Ascorbata in a pregnant woman. $J$ Coll Physicians Surg Pak 2020; 30(3):324-326.

\section{INTRODUCTION}

Kluyvera was firstly defined by Kluver and van Neil in 1936, although its complete discovery and molecular characterisation were performed by Farmer et al. in 1981.1,2 During the first years after characterisation, Kluyvera was regarded as a part of normal flora of the respiratory system, gastrointestinal tract, and urinary tract. However, in the last three decades, Kluyvera genus has been associated with clinically significant and serious infections. Nevertheless, our knowledge about the clinical significance of this organism is limited to case reports and small case series. ${ }^{3-5}$

Among the Kluyvera species, $K$. ascorbata has been isolated from only a few adult cases. ${ }^{6,7}$ Furthermore, there is little or no information in the literature as to whether the species of Kluyvera can cause clinically significant infections in pregnant women. Herein, we report a case of severe infection (urosepsis) caused by $K$. ascorbata in a pregnant woman.

\section{CASE REPORT}

A 23-year pregnant woman at 26 weeks of gestation presented to our hospital with left flank pain, vomiting,

Correspondence to: Dr. Akif Erbin, Department of Urology,

Haseki Training and Research Hospital, Istanbul, Turkey

E-mail:akiferbin@hotmail.com

Received: June 03, 2019; Revised: August 04, 2019;

Accepted: August 22, 2019 and irritative lower urinary tract symptoms. Patient history indicated that the patient had spontaneous stone passage two years earlier and had no previous history of surgery and extracorporeal shock wave lithotripsy. On admission, her temperature was $37.3^{\circ} \mathrm{C}$, pulse rate was 88 beats/min, and blood pressure was $105 / 62 \mathrm{mmHg}$. Total white blood cell (WBC) count was $7.9 \times 10^{3}$ cu.mm or $\mathrm{mm} 3$ and the hemoglobin was $10.4 \mathrm{~g} / \mathrm{dL}$. Urinalysis showed large amounts of red cells with no suspicion of urinary tract infection. Urinary ultrasonography showed left grade 3 hydronephrosis, ureteral dilatation, and a 10-mm distal ureteral stone (Figure 1). The patient was hospitalised and palliative treatment was initiated. However, the pain was not completely cured despite adequate medical treatment; and thus she underwent left ureterorenoscopy and laser lithotripsy. At the end of the procedure, a JJ stent were inserted and the patient

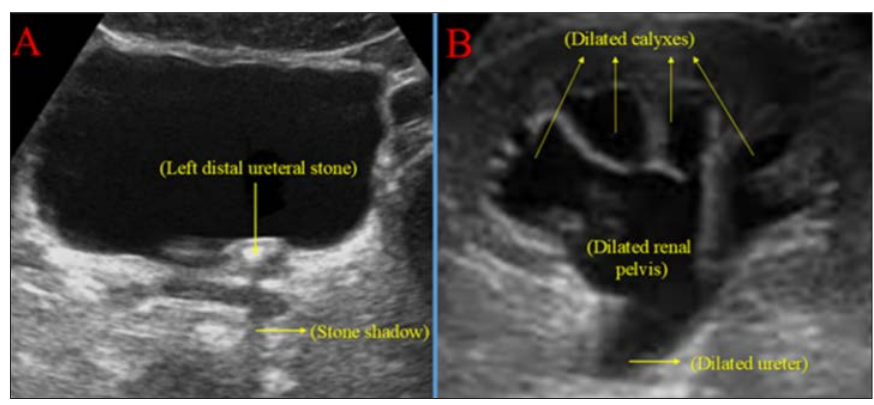

Figure 1: (A) Ultrasound image showing $10 \mathrm{~mm}$-left distal ureteral stone. (B) Ultrasound image showing grade-3 hydronephrosis and ureteral dilatation. 
was discharged on the second day. The pre-procedure culture was sterile.

Ten days later, the patient was readmitted to hospital with the complaints of nausea, vomiting, dizziness, and fever for two days. Physical examination revealed left costovertebral angle tenderness. The patient appeared toxic and the temperature, pulse rate, respiratory rate, and blood pressure were $39.8^{\circ} \mathrm{C}, 120$ beats/min, 32 breaths/min, and 95/52 mmHg, respectively. Preliminary laboratory results showed significant leukocytosis with a WBC count of $18.1 \times 10^{3}$ cu.mm or $\mathrm{mm}^{3}$, elevated C-reactive protein (CRP) of $146 \mathrm{mg} / \mathrm{L}$, and hyponatremia (130 mmol/dL). On urine analysis, large amounts of red and white cells and multiple bacteria were detected. Accordingly, the patient was diagnosed as having urosepsis. After blood and urine sampling, empirical antibiotherapy (ceftriaxone $2 \mathrm{~g} /$ day) and intravenous (IV) hydration were initiated. The blood pressure responded to IV fluid boluses. On third day of treatment, blood culture was sterile and $K$. ascorbata growth $\left(1 \times 10^{5}\right.$ $\mathrm{cfu} / \mathrm{mL}$ ) was detected in urine culture. Based on the clinical status of the patient and the antimicrobial susceptibility testing, the treatment was switched to ertapenem $1 \times 1 \mathrm{~g} /$ day and was continued for 14 days. Her fever and general clinical status responded well to treatment; and a repeat urine culture was negative for growth. The JJ stent was removed and the patient was discharged on the $17^{\text {th }}$ day of hospitalisation.

\section{DISCUSSION}

As a common saprophytic organism, Kluyvera is widely destributed in the environment such as in soil, water and sewage. It is also a member of the normal human gastrointestinal, urinary, and respiratory system flora. Although it was previously known as a commensal microorganism, Kluyvera has been recently shown to be pathogenic in humans and to cause clinically significant infections. There are three main pathogenic species of Kluyvera: K. ascorbata, K. cryocrescensa and K. species group. $3,6,7$

Kluyvera can cause clinical infections in humans such as gastroenteritis, cholecystitis, peritonitis, soft tissue abscesses, central nervous catheter infections, urinary tract infections, pyelonephritis, and sepsis. Moreover, it mostly affects immunocompromised patients, although immunocompetent individuals may also be affected. Neonates are at increased risk for Kluyvera infections, probably due to their immature immune system. Another important risk factor is having a chronic disease or an underlying predisposing condition.6-8 Among the Kluyvera species, $K$. ascorbata is the most common pathogen causing clinical infections in humans. The most common clinical presentations are urinary tract infections and pyelonephritis. Neonates and immunocompromised individuals are candidates of $K$. ascorbata infection.9,10 Moreover, $K$. ascorbata may also cause urinary tract infections in immunocompetent individuals, though rarely. 6-8

To the authors knowledge, lower urinary tract infection due to $K$. ascorbata has been reported only in a 18-year old pregnant patient. ${ }^{11}$ However, we could not find any study reporting on severe urinary infections (pyelonephritis or urosepsis) caused by $K$. ascorbata in pregnancy. 6 Pregnancy is a predisposing factor for both urinary tract infection and urinary stone formation. ${ }^{11}$ In particular, $K$. ascorbata becomes pathogenic in certain conditions such as bacterial overgrowth and increased virulence. This microorganism may translocate from the gastrointestinal flora to the blood stream, leading to bacteremia and localisation in distant sites. Genital infections as well as profound antibiotic and contraceptive use impair the normal perineal flora in females and prepare a suitable medium for the colonisation of $K$. ascorbata and other gram-negative organisms. ${ }^{7}$ This pathophysiologic mechanism seems to be appropriate for our patient, mainly because the patient did not have immunodeficieny or any chronic disease and did not get any immunosuppresive treatment, such as steroid therapy. However, pregnancy itself can be regarded as a relative immunosuppresive condition that may cause urinary tract infection in previosly healthy females. ${ }^{12}$

In conlcusion, Kluyvera genus may result in clinically significant infections despite being an infrequent pathogen. Additionally, it can cause life-threatening infections particularly in children, immunocompromised patients, and pregnant women. Therefore, clinicians should be aware of the potential risks of Kluyvera species. Among the Kluyvera species, $K$. ascorbata is the most frequent pathogen which can be isolated from pregnant women and may cause severe infection (urosepsis) during pregnancy. Accordingly, K. ascorbata should be considered in the differential diagnosis of pregnant patients presenting with urinary system-related urosepsis.

\section{PATIENT'S CONSENT:}

A written informed consent was obtained from the patient.

\section{CONFLICT OF INTEREST:}

Authors declared no conflict of interest.

\section{AUTHORS' CONTRIBUTION:}

$A E$ : Conception, design, literature research and manuscript writing.

HTG, YG: Interpretation of data and revising.

HLC: Interpretation of data and revising, supervision of the final version, English redaction.

\section{REFERENCES}

1. Kluyver AJ, van Niel CB. Prospects for a natural system of classification of bacteria. Zentralbl Bakteriol Parasitenkd Infektionskr Hyg 1936; 95:369-403. 
2. Farmer JJ, Fanning GR, Huntley-Carter GP, Holmes B, Hickman FW, Richard C, et al. Kluyvera, a new (redefined) genus in the family Enterobacteriaceae: Identification of Kluyvera ascorbata sp. nov and Kluyvera cryocrescens sp. nov. in clinical specimens. J Clin Microbiol 1981; 13:919-33.

3. Carter JE, Evans TN. Clinically significant Kluyvera infections: A report of seven cases. Am J Clin Pathol 2005; 123:334-8.

4. West BC, Vijayan H, Shekar R. Kluyvera cryocrescens finger infection: Case report and review of eighteen Kluyvera infections in human beings. Diagn Microbiol Infect Dis 1998; 32:237-41.

5. Karadag Öncel E, Özsürekci $Y$, Akyön $Y$, Gür D, Cengiz AB, Kara A. Kluyvera ascorbata infections in children: A case series. Turk Pediatri Ars 2015; 50:123-8.

6. Sarria JC, Vidal AM, Kimbrough RC. Infections caused by Kluyvera species in humans. Clin Infect Dis 2001; 33:69-74.

7. Torre D, Crespi E, Bernasconi M, Rapazzini P. Urinary tract infection caused by Kluyvera ascorbata in an immuno- compromised patient: Case report and review. Scand J Infect Dis 2005; 37:375-8.

8. Isozaki A, Shirai K, Mimura S, Takahashi M, Furushima W, Kawano $Y$. A case of urinary tract infection caused by Kluyvera ascorbata in an infant: Case report and review of the literature. $J$ Infect Chemother 2010; 16:436-8.

9. Sharma D, Dasi T, Murki S, Oleti TP. Kluyvera ascorbata sepsis in an extremely low birth weight infant. Indian J Med Microbiol 2015; 33:437-9.

10. Alfreijat M. A case of urinary tract infection and severe sepsis caused by kluyvera ascorbata in a 73-year-old female with a brief literature review. Case Rep Infect Dis 2017; 2017: 3848963.

11. Gozdas HT, Dinek M. Persistent fever during treatment of a pregnant woman with acute pyelonephritis. J Acute Dis 2017; 6:41-2.

12. Kourtis AP, Read JS, Jamieson DJ. Pregnancy and infection. N Engl J Med 2014; 370:2211-8. 